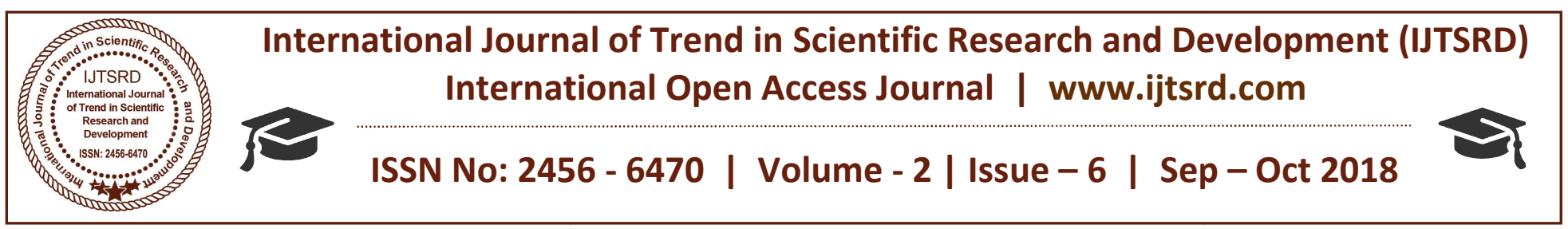

\title{
Performance Analysis of PV Modules: A Review
}

\author{
Trupti L. Chavan \\ Department of Electronics \& Communication Technology, \\ Shivaji University, Kolhapur, India
}

\begin{abstract}
In this paper literature review of performance analysis of PV modules are supposed to be monitored continuously for their parameters open voltage (Voc), short current (Isc), temperature, insolation, time etc. In proposed system don't need any person to be present physically on sight, so that the real time monitoring system is used. Home automation unit, sports using wireless sensor network. This monitoring system use for check PV modules performance analysis daily. Different platform like Microcontroller, ASIC, PIC microcontroller and embedded systems are used to design the system based on this performance and in the recent years cloud based systems have emerged. In future using IoT we can develop a system which will help to monitor different PV panel parameters or Faults.
\end{abstract}

Keywords: PV module, Time Monitoring, Wireless Sensor Network.

\section{INTRODUCTION}

In today's life PV energy is an important source of renewable energy and its technologies broadly classified either passive or active solar depending on how they capture and distribute solar energy or convert it into solar power. Active techniques are the use of Photovoltaic systems, concentrated solar power and solar water heating to harness the energy. Passive techniques are orienting a building to the Sun, selecting materials with favourable thermal mass or light-dispersing properties, and designing spaces that naturally circulate air. We can create such insights using only a smart way of generating, monitoring and distributing. Quality performance monitoring should be an essential component of PV energy system, but it is getting sacrificed in the name of cost-savings. Intelligent monitoring \& controlling analysis, controlling and tracking energy generation to analyse performance of the system.

The PV systems are expected to produce a certain amount of energy during each day. A solar monitoring $\&$ controlling system can inform if the system are off line or if it is not functioning as it was expected in order to take actions or run diagnostic programs. A solar PV plant where the parameters must be closely monitored and controlled thus requires an adequate data acquisition system (DAQ). The data acquisition system requires a large number of measured data where very frequent recordings need to be automated to eliminate the probability of human error as well as to save time. It is also necessary that the data can be represented in a graphical form for straight forward monitoring and analysis compare from having the data in a numerical format to achieve desire performance. And create GUI for same.

In general, IoT is actually an information sharing environment where objects in every-day life are connected to wired and wireless networks. Recently, it is used not only for the field of consumer electronics and appliances but also in other various fields such as a smart city, healthcare, smart home, smart car, energy system, and industrial security. At present, the photovoltaic (PV) energy is one of the important renewable energy sources. The PV energy is becoming a potential solution towards sustainable energy supply in future. As more and more Rooftop Solar Photovoltaic systems are getting integrated into the existing grid, there is a growing need for monitoring of real time generation data obtained from solar photovoltaic plants so as to optimize the overall performance of the solar and to maintain the stability and reliability.

Power generation from Solar Photovoltaic plants is variable in nature due to changes in solar irradiance, temperature and insolation and other factors. Thus monitoring is essential for developing monitoring 
system for photovoltaic module, IoT (Internet of Things) approach is taken in this work which actually envisions a near future where everyday objects will be armed with microcontrollers and transceivers for digital communication. The monitoring eliminate the hazards associated with the traditional wiring systems and make data measurement and monitoring process much easier and cost effective and IoT based systems take a giant leap towards monitoring by intelligent decision making. The architecture of monitoring systems and its flexibility of deployment make it most suitable for industrial purposes.

\section{LITERATURE REVIEW}

Several development of performance analysis of PV modules. Also there are several implementation of Fault Detection of PV modules using the IoT and Wireless/wired Networks. Also there are novel implementations of Solar PV modules monitoring solar PV power conditioning unit Fault Detection, Home Appliances, Maintenance. Work done in this regard by few researchers is discussed here.

The smart solar photovoltaic remote monitoring and control unit using the Internet of Things Technology is implemented [1]. With advancement 1C of technologies the cost of renewable energy equipment's is going down globally encouraging large scale solar photovoltaic installations. This massive scale of solar photovoltaic deployment requires sophisticated systems for automation of the plant monitoring remotely using web based interfaces as majority of them are installed in inaccessible locations and thus unable to be monitored from a dedicated location. The discussion in this paper is based on implementation of new cost effective methodology based on IoT to monitor a solar photovoltaic plant for performance evaluation. This will facilitate preventive maintenance, fault detection, historical analysis of the plant in addition to real time monitoring.

For Monitoring Solar PV Power Conditioning Unit based on IOT System is designed [2].An effective implementation of an intelligent remote monitoring system for Photovoltaic (PV) Power Conditioning Unit which is used in a greenhouse environment. The proposed system design can be installed in solar PCU in order to solve management problems, maintenance and shortens the mean time to repair. They have designed a smart remote monitoring system based on internet of thing for monitoring Solar PCU. This system had incorporated remote monitoring for solar
PCU through internet using host, network Global Positioning Radio Service, embedded system gateway and other components. The result of our demonstration shows the system can monitor store and manipulate data from solar PCU. Thus, the remote monitoring functions are realized in real-time system.

Automated Controlling Software System and Intelligent Monitoring system for solar energy is implemented [3]. The inspection of the solar panels on a periodic basis is important to improve performance of the solar power system. To get the more solar energy of the photovoltaic (PV) system is possible through an intelligent monitoring system. The monitoring system has rapidly increased its popularity because of its user friendly graphical interface for data acquisition, monitoring, controlling and measurements. In order to monitor the performance of the system especially for renewable energy source application such as photovoltaic, dataacquisition systems had been used to collect all the data regarding the installed system. The development of a smart automated monitoring \& controlling system for the solar panel is described, the core idea is based on IoT (the Internet of Things). The advantages of the system are the performance of the PV panel system which can be monitored and analysed.

Automatic detection checking of Solar Panels Using Internet of Things is developed [4]. Examine and checking faults on a set of Solar panels, using Internet of Things. An effective implementation of remote observing system for solar Photovoltaic cell (PV) and Power Conditioning Unit. Today, with the growth in sensor technology it is a very likely option to connect the PV energy systems to the cloud (internet) with the help of Internet of Things, the analysis of the performance, productivity and efficiency can be calculated very easily when system is connected to cloud. With the software-technology monitoring of vast PV panels are made easy and accurate. In output of system the data are monitored manually and noted down in notebooks and excel file. The sensors such as temperature sensors are fixed on the PV panels and the current voltage produced are been transmitted through wireless communication and monitor in base station (system).

Based on IoT the Smart Monitoring and Controlling of PV System is implemented [5]. In order to analyse the performance of photovoltaic systems, they have 
developed a real-time expert system based on a central microcomputer used as a micro server and can be easily consulted from different automatic stations. The developed system is able to ensure the monitoring, supervision, and control of PV systems installed over a wide area, on one hand, and to create a general PV systems database, on the other. This system presents a design of a universal data acquisition system with available components and which is easily accessible through a server. This system presents a novel procedure for fault diagnosis in PV systems with Internet of Things (IoT).This work describes the development of a system designed for power generation integration. It continuously acquires solar and temperature, current, voltage, irradiance which is automatically correlated with energy parameters, obtained from renewable energy systems. The developed system was installed in a photovoltaic power generation. The developed application allows, in addition to the acquisition of weather and energy data, their continuous monitoring and correlation through a graphical user interface, providing a friendly interactivity with the user (5).

Monitoring of Solar PV cell Performance with Changing Irradiance and Temperature Solar energy is most readily available source of energy is designed [6]. It is Non-polluting and maintenance free. To make best use of the solar PV systems the output is maximized either by mechanically tracking the sun and orienting the panel in such a direction so as to receive the maximum solar irradiance or by electrically tracking the maximum power point under changing condition of insolation and temperature. The overall performance of solar cell varies with varying Irradiance and Temperature .With the change in the time of the day the power received from the Sun by the PV panel changes. Not only this both irradiance and temperature affect PV cell efficiency as well as corresponding Fill factor also changes. This paper gives an idea about how the solar cell performance changes with the change in above mentioned factors in reality and the result is shown by conducting a number of experiments.

The detection of Photovoltaic Systems for Performance and Fault Identification is designed [7]. The stability of standalone photovoltaic systems passes through an addition of the systems installed in the field. This addition had been developed procedures for monitoring a similar system in laboratory. The standalone photovoltaic system
Implanted in the Research Centre in Intelligent Energy of the Group of Studies in Energy - CPEI GREEN PUC Minas is similar to the systems installed by Campania Energetic de Minas Cereals - CEMIG in the schools of isolated communities, inside the SOLAR LIGHT Program. A simulation of the system was implemented and the aims were to optimize the project and carry out a comparative study with the monitoring results. The procedure for assembly of the monitoring facility consisted of the implantation of the voltage and current sensors, implantation of the irradiance and temperature sensors, installation of the acquisition boards and development of the monitoring program. The results presented here will allow the development of a program of preventive maintenance of the photovoltaic systems installed.

Online Fault Detection in solar panel systems is development [8] of a practical fault detection approach in photovoltaic (PV) systems, intended for online implementation. It was developed and validated using field measurements from a Canadian PV system. It has a fairly low complexity, but achieves a high fault detection rate and is able to successfully cope with abnormalities present in reallife measurements. The fault detection is based on the comparison between the measured and model prediction results of the ac power production. The model estimates the ac power production using solar irradiance and PV panel temperature measurements. Prior to model development, a data analysis procedure was used to identify values not representative of a normal PV system operation. The original 10-min measurements were averaged over $1 \mathrm{~h}$, and both datasets were used for modelling. In order to better represent the PV system performance at different sunlight levels, models for different irradiance ranges were developed. The results reveal that the models based on hourly averages are more accurate than the models using 10-min measurements, and the models for different irradiance intervals lead to a fault detection rate greater than $90 \%$.

The PV system performance ratio (PR) was used to keep track of the system's long-term performance.

\section{CONCLUSION}

An overview of performance analysis of PV module it seems that less work has been carried out on performance assessment of PV modules in manufacturing industry. Much work focuses on condition monitoring of PV module on site where the 
PV arrays are installed (power generation stations). Many researchers have worked on performance study of PV module using wireless sensor networks, simulation tools etc. In PV module manufacturing industry performance assessment is significant task and many times it is carried out manually, where team of people is assigned task of characterization of PV manufactured modules. This could involve error as the task heavily depends upon accuracy provided by technicians. At few manufacturing industries artificially insolation is created and atmospheric parameters are controlled for PV module performance testing. But this method does not provide real time performance assessment; for this we can performance of multiple PV modules will be assessed in real time in real environment. In this facility will be provided to online assess the performance of multiple PV modules by using IoT. The system developed here will be user friendly.

\section{REFERENCES}

1. Soham Adhya1, Dipak Saha2, Abhijit Das3, Joy dip Jana4, Hiranmay Sa, "An IoT Based Smart Solar Photovoltaic Remote Monitoring and Control unit" 2016 2nd International Conference on Control, Instrumentation, Energy \& Communication (CIEC)

2. Shrihariprasath.B, Dr. Vimalathithan Rathinasabapathy "A Smart IoT System/for Monitoring Solar PV Power Conditioning Unit" 2016 World Conference on Futuristic Trends in Research and Innovation for Social Welfare (WCFTR'16)

3. H S Nalamwar, M A Ivanov and S A Baidali "Automated Intelligent Monitoring and the Controlling Software System for Solar Panels" International Conference on Information Technologies in Business and Industry 2016

4. R. Vignesh, Dr.A.Samydurai "Automatic Monitoring and Lifetime Detection of Solar Panels Using Internet of Things" International Journal of Innovative Research in Computer and Communication Engineering Vol. 5, Issue 4, April 2017

5. M. Latha, S. Mani, R. Manikandan, "Smart Monitoring and Controlling of PV System Using IoT" International Journal of Modern Science and Technology Vol. 2, No. 4, 2017.

6. Pradhan Arjyadhara, Ali S.M, Jena Chitralekha, "Analysis of Solar PV cell
Performance with Changing Irradiance and

Temperature" International Journal Of Engineering And Computer Science ISSN:2319-7242 Volume 2 Issue 1 Jan 2013.

7. L. de V. B. Machado Neto, C. V. T. Cabral, D. Oliveira Filho, and A, S. A. C. Dink" Monitoring of Photovoltaic Systems for Performance Evaluation and Fault Identification" 2004 IEEWPES Transmission \& Distribution Conference \& Exposition: Latin America

8. Radu Platon, Jacques Martel, Norris Woodruff, and Tak Y. Chau, "Online Fault Detection in PV Systems" IEEE Transactions on Sustainable Energy Issue Date: October 2015 Volume: 6 Issue: 4

9. Xi Chen, Limin Sun ; Hongsong Zhu ; Yan Zhen ; Hongbin Chen, "Application of Internet of Things in Power-Line Monitoring",2012 International Conference on Cyber-Enabled Distributed Computing and Knowledge Discovery (CyberC),978-1-4673-2624-7.

10. Byeongkwan Kang, Sunghoi Park, Tacklim Lee, and Sehyun Park," IoT-based Monitoring System using Tri-level Context Making Model for Smart Home Services", 2015 IEEE International Conference on Consumer Electronics (ICCE).

11. Achim Woyte, Mauricio Richter, David Moser, Stefan Mau, Nils Reich, Ulrike Jahn "Monitoring Of Photovoltaic Systems: Good Practices and Systematic Analysis" 28th European PV Solar.

12. Suciu Constantin, Florin Moldoveanu, Radu Campeanu, Ioana Baciu, Sorin Mihai Grigorescu, Bogdan Carstea, Vlad Voinea, "GPRS Based System for Atmospheric Pollution Monitoring and Warning", 1-42440361-8/06/\$20.00 C2006 IEEE.

13. Chen Peijiang, Jiang Xuehua,"Design and Implementation of Remote Monitoring System Based on GSM", 2008 IEEE Pacific-Asia Workshop on Computational Intelligence and Industrial Application.

14. Oussama BEN BELGITH, Lasaad SBITA, "Remote GSM module monitoring and Photovoltaic System control”, 2014 First International Conference on Green Energy ICGE 2014. 
International Journal of Trend in Scientific Research and Development (IJTSRD) ISSN: 2456-6470

15. Qinghai Ou, Yan Zhen, Xiangzhen Li, Yiying Zhang, Ling kang Zeng, "Application of Internet of Things in SmartGrid Power Transmission",2012 Third FTRA International Conference on Mobile, Ubiquitous, and Intelligent Computing.

16. Bakos G C 2009 Distributed power generation: A case study of small scale PV power plant in Greece Applied Energy 86 1757-1766

17. Iacob M, Andree cu G D, and Muntean N 2009 SCADA System for a Central Heating and Power Plant 5th International Symposium on applied Computational Intelligence and Informatics 159-164
18. Chan F, Calleja H 2006 Reliability: A New Approach Design of Inverters for PV Systems 10th IEEE International Power Electronics Congress 2006 498-506

19. Hamoud, Chen G, Bradley R L 2003 Risk Assessment of Power Systems Performance Monitoring IEEE Transactions on Instrumentation and Measurement 2

20. Wang H, Hu S 2009 Energy Saving Analysis of Supervisory Control and Data Acquisition System Applied in District Heating ICIEA 3245-3247. 